This article was published on 9 Oct 2020 at www.hkmj.org.

\title{
Povidone-iodine and carrageenan are candidates for SARS-CoV-2 infection control
}

\author{
Hong Kong Med J 2020;26:464 \\ https://doi.org/10.12809/hkmj208889
}

To the Editor-Povidone-iodine, a candidate for nasal spray and oral rinse, has displayed viricidal effects on severe acute respiratory syndrome coronavirus 2 (SARS-CoV-2) in vitro after 30 to $60 \mathrm{~s}$ of exposure, even at concentrations as low as $0.45 \%{ }^{1}$ International dental and endodontics associations have suggested povidone-iodine as a preprocedural mouthrinse to limit the spread of SARS-CoV-2. ${ }^{2}$ In a Letter to the Editor of Oral Oncology, Mady et $\mathrm{al}^{3}$ recommend nasal and oral povidone-iodine in head and neck oncology patients and healthcare providers every 2 to 3 hours, with a maximum of 4 times per day to mitigate SARS-CoV-2 transmission. It is noteworthy that hypo- and hyper-thyroidism are possible adverse effects and contra-indications of povidone-iodine use. ${ }^{4}$ Nevertheless, such adverse effects are infrequent, and occur primarily after long-term use (eg, >1 year). ${ }^{4}$ Povidone-iodine is also contra-indicated with breastfeeding and pregnancy due to the occurrence of neonatal hypothyroidism. ${ }^{5}$ After excluding contra-indications, povidoneiodine mouthrinse or oral/nasal spray can reduce SARS-CoV-2 viral load in the upper respiratory tract, hampering transmission when used in conjunction with existing infection control or public health measures.

In addition to povidone-iodine, carrageenan in over-the-counter lozenges or nasal spray may be an appropriate candidate. As a sulphated polysaccharide which cannot penetrate the mucosal membrane, it acts through physically inhibiting viral attachment and entry into host cells. ${ }^{6,7}$ Such non-specific mechanism explains the broad viricidal activity of nasal spray against enveloped (eg, influenza and coronavirus) and non-enveloped viruses (eg, rhinovirus) in vivo, with a greater reduction of disease duration and likelihood of relapse among coronavirus patients. ${ }^{7}$ The latest evidence further proves the tight binding of sulphated polysaccharide to the spike protein of SARS-CoV-2. ${ }^{8}$ With low cytotoxicity and as a food additive classified as "Generally Recognised as Safe", carrageenan nasal sprays demonstrate high tolerability. ${ }^{6-8}$

In conclusion, povidone-iodine and carrageenan possess potential for use as chemoprophylaxis or even in adjunct with the current health behaviours (hand hygiene, face mask use) and social distancing measures to synergistically suppress SARS-CoV-2 transmission.

\section{Author contributions}

The author drafted the letter. The author approved the final version for publication and takes responsibility for its accuracy and integrity.

\section{Conflicts of interest}

The author has disclosed no conflicts of interest.

\section{Funding/support}

This letter received no specific grant from any funding agency in the public, commercial, or not-for-profit sectors.

KK Hui *, BSc

Faculty of Medicine, The Chinese University of Hong Kong, Hong Kong

* Corresponding author: kegankkhui@link.cuhk.edu.hk

\section{References}

1. Anderson DE, Sivalingam V, Kang AE, et al. Povidoneiodine demonstrates rapid in-vitro virucidal activity against SARS-CoV-2, the virus causing COVID-19 disease. Infect Dis Ther 2020;9:669-75.

2. Jamal M, Shah M, Almarzooqi SH, et al. Overview of transnational recommendations for COVID-19 transmission control in dental care settings. Oral Dis 2020 May 19. Epub ahead of print.

3. Mady LJ, Kubik MW, Baddour K, Snyderman CH, Rowan NR. Consideration of povidone-iodine as a public health intervention for COVID-19: Utilization as "Personal Protective Equipment" for frontline providers exposed in high-risk head and neck and skull base oncology care. Oral Oncol 2020;105:104724.

4. Nobukuni K, Hayakawa N, Namba R, et al. The influence of long-term treatment with povidone-iodine on thyroid function. Dermatology 1997;195 Suppl 2:69-72.

5. Casteels K, Pünt S, Brämswig J. Transient neonatal hypothyroidism during breastfeeding after postnatal maternal topical iodine treatment. Eur J Pediatr 2000;159:716-7.

6. Eccles R, Winther B, Johnston SL, Robinson P, Trampisch M, Koelsch S. Efficacy and safety of iota-carrageenan nasal spray versus placebo in early treatment of the common cold in adults: the ICICC trial. Respir Res 2015;16:121.

7. Koenighofer M, Lion T, Bodenteich A, et al. Carrageenan nasal spray in virus confirmed common cold: individual patient data analysis of two randomized controlled trials. Multidiscip Respir Med 2014;9:57.

8. Kwon PS, Oh H, Kwon SJ, et al. Sulfated polysaccharides effectively inhibit SARS-CoV-2 in vitro. Cell Discov 2020;6:50. 